\title{
A Novel Cubic-Order Algorithm for Approximating Principal Direction Vectors
}

\author{
JACK GOLDFEATHER \\ Carleton College \\ and \\ VICTORIA INTERRANTE \\ University of Minnesota
}

\begin{abstract}
There are a number of applications in computer graphics that require as a first step the accurate estimation of principal direction vectors at arbitrary vertices on a triangulated surface. Although several methods for calculating principal directions over such models have been previously proposed, we have found in practice that all exhibit unexplained large errors in some cases. In this article, we describe our theoretical and experimental investigations into possible sources of errors in the approximation of principal direction vectors from triangular meshes, and suggest a new method for estimating principal directions that can yield better results under some circumstances.
\end{abstract}

Categories and Subject Descriptors: I.3.5 [Computer Graphics]: Computational Geometry and Object Modeling; I.3.7 [Computer Graphics]: Three-Dimensional Graphics and Realism

General Terms: Algorithms, Design, Experimentation

Additional Key Words and Phrases: 3D shape, principal directions

\section{INTRODUCTION}

Suppose we are given only a surface mesh of vertices and polygons approximating some unknown smooth surface. There have been many methods proposed for approximating principal directions of the underlying surface [Chen and Schmitt 1992; Flynn and J ain 1989; Hamann 1994; Meyer et al. 2003; Samson and Mallet 1997; Taubin 1995]. In this article, we will examine a few of the known methods, showing how well they can work in some cases and how badly they can fail in others. In particular, we will show how very tiny normal curvature approximation errors can be magnified intolargeerrors in the estimated principal directions. We also introduce a new method that we believe performs significantly better under certain conditions than many other proposed methods. In Section 2, we briefly describe the motivation for this work and its significance to applications in computer graphics. In Section 3, we review the basic mathematics behind computing principal directions, stating the necessary formulas

This work was supported in part by a grant from the National Science Foundation (NSF) ACI-9875368.

Authors' addresses: J. Goldfeather, Department of Mathematics and Computer Science, Carleton College, One North College Street, Northfield, MN 55057; email: jgoldfea@mathcs.carleton.edu. V. Interrante, Deprtment of Computer Science and Engineering, University of Minnesota, 4-192 EECS BIdg., 200 Union Street, SE, Minneapolis, MN 55455; email: interran@cs.umn.edu. Permission to make digital or hard copies of part or all of this work for personal or classroom use is granted without fee provided that copies are not made or distributed for profit or direct commercial advantage and that copies show this notice on the first page or initial screen of a display along with the full citation. Copyrights for components of this work owned by others than ACM must be honored. Abstracting with credit is permitted. To copy otherwise, to republish, to post on servers, to redistribute to lists, or to use any component of this work in other works requires prior specific permission and/or a fee. Permissions may be requested from Publications Dept., ACM, Inc., 1515 Broadway, New York, NY 10036 USA, fax: +1 (212) 869-0481, or permissions@acm.org. (C) 2004 ACM 0730-0301/04/0100-0045 $\$ 5.00$ 
for the Weingarten curvature matrix and for its use in computing normal curvature in a given direction. In Section 4, we describe in detail three methods each of which approximates the Weingarten curvature matrix at a vertex of the mesh. In Section 5, we apply each method to a test surface using a number of different mesh schemes and then examine the direction errors. In the course of our investigations, we probe the relationship between surface approximation errors and errors in principal directions, and find that similar-appearing surface approximation errors can lead to vastly different principal direction errors. In particular, we find that while the chance for large errors increases near umbilical points, they can still occur at points on the surface where there is significant difference between the two principal curvatures. We conclude by summarizing the conditions under which principal direction estimation errors are most likely to occur, suggesting some steps that can be taken to improve the situation, and outlining promising directions for future work in this area.

\section{MOTIVATION}

There are many applications for which it is useful to be able to calculate accurate estimates of the principal directions at points on a given surface. Principal direction vector fields have been widely used in shape analysis and surface interrogation in computer-aided manufacturing and design [Hahmann 1999; Takashi Maekawa and Patrikalakis 1996]. In these applications, the surfaces are analytically defined, so the principal directions can be solved for directly and there are few problems with errors in the estimations. Principal direction vector fields have also been successfully used in conjunction with volumetrically defined data to enhance the visual representation of surface shape for applications in molecular [Duncan and Olson 1992] and medical [I nterrante 1997] visualization. In these applications, the principal directions can be obtained by diagonalizing the Second Fundamental Form, whose entries can be fairly well approximated using first and second directional derivatives of the sampled 3D distribution.

Recently, there has been increasing interest expressed in the possibility of using principal direction vector fields over polygonal meshes for such purposes as guiding the direction of hatching strokes in pen-and-ink style renderings [Girshick et al. 2000; Hertzmann and Zorin 2000] or adaptively guiding the orientation of synthesized anisotropic texture patterns for enhanced surface shape representation [Diewald et al. 2000; Gorla et al. 2003]. However such efforts have been complicated by the lack of a robust and reliably accurate method for estimating the principal directions at points on an underlying smooth surface that is represented solely by a polygonal mesh. Although a number of methods for principal direction estimation have been previously published [Taubin 1995; Hamann 1994; M eyer et al. 2003; Flynn and J ain 1989; Chen and Schmitt 1992; Samson and Mallet 1997], what we have found in practice is that the computed vector field inevitably appears "noisy", requiring post hoc smoothing that can result in many of the vectors falling out of alignment with the true principal directions and complicating our efforts to generate surface markings that closely follow the form.

In the research described in this article, we sought to gain insight intothesources of errors in principal direction estimation on surfaces defined by point samples organized in a polygonal mesh, in order to determine what the worst potential pitfalls areand how they might be overcome. Our goal was to enable an approach in which instead of accepting large principal direction estimation errors and then working to hide them, we could strive to obtain more accurate initial principal direction estimates whileflagging the points at which the probability of obtaining erroneous estimates is high.

\section{A QUICK REVIEW OF SURFACE CURVATURE}

Let $p$ be a point on a smooth surface $S$, let $N_{p}$ be the unit normal to $S$ at $p$, and suppose $X(u, v)$ is a local parametrization of $S$ in a neighborhood of $p$. Then using $X_{u}(p), X_{v}(p), N_{p}$ as a local coordinate system, we can compute principal curvatures and principal directions as follows: Let $\lambda_{1}$ and $\lambda_{2}\left(\lambda_{1} \geq \lambda_{2}\right)$ 
be the eigenvalues, and $p_{1}, p_{2}$ the associated unit eigenvectors of the Weingarten curvature matrix

$$
W=\left(\begin{array}{ll}
\frac{e G-f F}{E G-F^{2}} & \frac{f E-e F}{E G-F^{2}} \\
\frac{f G-g F}{E G-F^{2}} & \frac{g E-f F}{E G-F^{2}}
\end{array}\right)
$$

where

$$
\begin{array}{ll}
e=N_{p} \cdot X_{u u}(p) & E=X_{u}(p) \cdot X_{u}(p) \\
f=N_{p} \cdot X_{u v}(p) & F=X_{u}(p) \cdot X_{v}(p) \\
g=N_{p} \cdot X_{v v}(p) & G=X_{v}(p) \cdot X_{v}(p)
\end{array}
$$

Note that in the special case that $X_{u}$ and $X_{v}$ are orthogonal unit vectors, this becomes the symmetric matrix

$$
W=\left(\begin{array}{ll}
e & f \\
f & g
\end{array}\right) .
$$

If $u$ is a unit vector in the tangent plane to $S$ at $p$, then

$$
\kappa_{\mathrm{u}}=\mathrm{u}^{\top} \mathrm{Wu}
$$

is the normal curvature of the surface in the direction of $u$.

It follows that $\lambda_{1}$ and $\lambda_{2}$ are the maximum and minimum normal curvatures of the surface at $p$, and $p_{1}=\left(\begin{array}{c}p_{11} \\ p_{12}\end{array}\right)$ and $p_{2}=\left(\begin{array}{c}p_{21} \\ p_{22}\end{array}\right)$ are the principal curvature vectors expressed in local coordinates. That is,

$$
\begin{aligned}
& v_{1}=p_{11} x_{u}+p_{12} X_{v} \\
& v_{2}=p_{21} x_{u}+p_{22} x_{v}
\end{aligned}
$$

are the principal direction vectors in $\mathrm{R}^{3}$.

An important observation is that at points where $\lambda_{1}=\lambda_{2}$, the notion of principal direction is not defined, since all vectors are eigenvectors. Such a point is called an umbilical point on the surface.

\section{THREE PRINCIPAL DIRECTION APPROXIMATION METHODS}

The first step in computing principal directions on a surface mesh is to compute at each vertex pa vector $\mathrm{N}_{\mathrm{p}}^{\prime}$ that approximates the true unit surface normal $\mathrm{N}_{\mathrm{p}}$ at $\mathrm{p}$. Most methods compute a "normalized average"- that is, a set of vectors is summed and the resulting vector is normalized to length 1.

Of course, using an incorrect surface normal will introduce error into a principal direction calculation. However, as we will show, even if the exact surface normal is used, there are other sources of significant potential error in principal direction estimation. In order to most effectively gain insight into these other sources of error, we decided to use exact surface normals in the subsequent steps of our initial investigations. Later, we return to examine the effects of using approximated surface normals.

The next step in estimating the principal directions is to choose a pair of orthonormal vectors $\mathbf{x}_{1}$ and $\mathbf{x}_{2}$ in the plane through $\mathrm{p}$ with normal vector $\mathrm{N}_{\mathrm{p}}^{\prime}$ to form a local orthonormal coordinate system $\mathrm{L}=\left\{\mathbf{x}_{1}, \mathbf{x}_{2}, \mathrm{~N}_{\mathrm{p}}^{\prime}\right\}$ in $\mathrm{R}^{3}$. All subsequent calculations are done with respect to this local coordinate system. We examined three principal direction estimation methods, outlined below, each of which approximates the Weingarten curvature matrix expressed in this local coordinate system. 


\subsection{The Normal Curvature Approximation Method}

Suppose $W$ is the unknown Weingarten matrix with respect to local coordinates $L$ at vertex $p$. Suppose there are $n$ vertices adjacent to $p$ and let $q_{i}$ denote the ith adjacent vertex. We denote by $\mathbf{y}_{i}$ the unit vector obtained by projecting the vector $\bar{p}_{i}$ (expressed in local coordinates $L$ ) onto the plane through $\mathrm{p}$ with normal vector $\mathrm{N}_{\mathrm{p}}^{\prime}$ and normalizing the result. Then using the result from Section 1 , the normal curvature in the direction $\mathbf{y}_{\mathrm{i}}$ is given by $\kappa_{\mathbf{y}_{i}}=\mathbf{y}_{\mathrm{i}}^{\top} \mathbf{W} \mathbf{y}_{\mathrm{i}}$. An approximation to this normal curvature is given by:

$$
\kappa_{\mathbf{y}_{i}}^{\prime}=2 \frac{\left(p-q_{i}\right) \cdot N_{p}^{\prime}}{\left(p-q_{i}\right) \cdot\left(p-q_{i}\right)},
$$

which is the curvature of the unique osculating circle passing through $p$ and $q_{i}$ with normal $N_{p}^{\prime}$ at $p$. This produces a system of equations:

$$
\mathbf{y}_{\mathrm{i}}^{\top} \mathrm{W} \mathbf{y}_{\mathrm{i}}=\kappa_{\mathbf{y}_{\mathrm{i}}}^{\prime} \quad \mathrm{i}=1,2, \ldots, \mathrm{n}
$$

that we wish to solve for W. In Meyer et al. [2003], these equations are weighted in the same manner as the weighting in the third normal approximation method mentioned at the beginning of this section, but it is not clear to us that this improves the result much. The problem is that the osculating circle only produces a second-order approximation to the true normal curvature and second-order approximations can introduce significant error in many cases.

The first step in solving for $W$ is to reorganize (1) as follows. Let $\mathbf{y}_{i}=\left(u_{i}, v_{i}\right)$ and

$$
W=\left(\begin{array}{ll}
A & B \\
B & C
\end{array}\right) .
$$

Then

$$
\mathbf{y}_{i}^{\top} W \mathbf{y}_{i}=\left(\begin{array}{ll}
u_{i} & v_{i}
\end{array}\right)\left(\begin{array}{cc}
A & B \\
B & C
\end{array}\right)\left(\begin{array}{l}
u_{i} \\
v_{i}
\end{array}\right)=\left(\begin{array}{lll}
u_{i}^{2} & 2 u_{i} v_{i} & v_{i}^{2}
\end{array}\right)\left(\begin{array}{c}
A \\
B \\
C
\end{array}\right) .
$$

If we let $U$ be the $n \times 3$ matrix with rows $\left(\begin{array}{llll}u_{i}^{2} & 2 u_{i} v_{i} & v_{i}^{2}\end{array}\right), \mathbf{x}=\left(\begin{array}{lll}A & B & C\end{array}\right)^{\top}$, and $d$ be the $n$-vector whose ith entry is $\kappa_{\mathbf{y}_{\mathbf{i}}}^{\prime}$ the entire system can be written as the matrix equation

$$
U \mathbf{x}=\mathbf{d} \text {. }
$$

If $\mathbf{d}$ was the vector of true normal curvatures, this linear system would have an exact solution (assuming the adjacent vertices do not have some degenerate pattern, like all lying on the same line.) In practice, the best we can hopeto find is a least squares fit, that is, a vector $\mathbf{x}$ that minimizes $\|\mathbf{U} \mathbf{x}-\mathbf{d}\|$. There are standard numerical methods for finding this least squares solution. The resulting matrix

$$
W^{\prime}=\left(\begin{array}{ll}
A^{\prime} & B^{\prime} \\
B^{\prime} & C^{\prime}
\end{array}\right)
$$

is used to approximate the principal directions. It is worth noting that the matrix $U$ depends only on the projection of the adjacent vertices onto the local tangent plane, that is, it is fixed by the choice of the mesh on the surface. The vector $\mathbf{d}$ depends on measurements we make at these adjacent vertices-that is, it varies in accordance with the amount of error we make. As we will see in a later section, it is possible to separate the mesh from the measurements so that we can understand how measurement error interacts with the local mesh geometry. 
4.2 The Quadratic Surface Approximation Method

In this method, we try to best-fit a quadratic surface to the adjacent vertices. We begin by transforming each adjacent vertex $q_{i}$ to local coordinates $\left(x_{i}, y_{i}, z_{i}\right)$. In these local coordinates, p becomes $(0,0,0), N_{p}^{\prime}$ lies along the positive $z$-axis, and the quadratic surface looks like

$$
z=f(x, y)=\frac{A}{2} x^{2}+B x y+\frac{C}{2} y^{2} .
$$

It is easy to show that the Weingarten matrix for such a surface is

$$
W=\left(\begin{array}{ll}
A & B \\
B & C
\end{array}\right)
$$

As in the Normal Curvature method, we plug in the adjacent vertices to get a system of equations

$$
\left(\frac{1}{2} x_{i}^{2} \quad x_{i} y_{i} \quad \frac{1}{2} y_{i}^{2}\right) x=z_{i} \quad i=1, \ldots, n .
$$

As before, we can find a least-squares fit to this system. In order to be able to compare the result to the normal curvature method, we scale the ith equation by $\frac{2}{k_{i}^{2}}$, where $k_{i}=\sqrt{x_{i}^{2}+y_{i}^{2}}$. Then $\left(x_{i}, y_{i}\right)=k_{i}\left(u_{i}, v_{i}\right)$ so that

$$
\left(\begin{array}{lll}
\frac{1}{2} x_{i}^{2} & x_{i} y_{i} & \frac{1}{2} y_{i}^{2}
\end{array}\right)=\frac{k_{i}^{2}}{2}\left(\begin{array}{lll}
u_{i}^{2} & 2 u_{i} v_{i} & v_{i}^{2}
\end{array}\right) .
$$

Then, we can write each scaled equation in the system as:

$$
\left(\begin{array}{lll}
u_{i}^{2} & 2 u_{i} v_{i} & v_{i}^{2}
\end{array}\right) \mathbf{x}=d_{i},
$$

where $d_{i}=\frac{2}{k_{i}^{2}} z_{i}$. There is a nice geometric interpreatation of $d_{i}$. Consider the parabola with equation $y=\frac{d_{i}}{2} x^{2}$. Plugging in $x=k_{i}$ we get

$$
y=\frac{d_{i}}{2} k_{i}^{2}=z_{i},
$$

so that this parabola passes through the origin and the point $\left(k_{i}, z_{i}\right)$. Since $k_{i}=\sqrt{x_{i}^{2}+y_{i}^{2}}$, we can think of this parabola in three dimensions as passing through thelocal coordinate origin and the point $\left(\mathrm{x}_{\mathrm{i}}, \mathrm{y}_{\mathrm{i}}, \mathrm{z}_{\mathrm{i}}\right)$.

The one variable, planar formula for computing curvature, is :

$$
\kappa=\frac{\left|\mathrm{y}^{\prime \prime}\right|}{\left(1+\mathrm{y}^{\prime 2}\right)^{3 / 2}} .
$$

Using this formula for the parabola, at $(0,0)$ we get $\kappa=\left|d_{i}\right|$. In other words, $d_{i}$ is the normal curvature of a parabola passing through $\left(\mathrm{x}_{\mathrm{i}}, \mathrm{y}_{\mathrm{i}}, \mathrm{z}_{\mathrm{i}}\right)$ and the origin. We can now see that the Quadratic Surface method is identical to the Normal Curvature method, except that curvature approximations are done using parabolas rather than circles. This suggests that these two methods will produce similar results, with one performing slightly better than the other depending on whether circles or parabolas better approximate the surface locally in some direction.

\subsection{The Adjacent-Normal Cubic Approximation Method}

In both of the proceeding methods, we did not use all of the information available to us. Namely, we did not use the known (approximated) normal vectors at adjacent vertices $\mathrm{q}_{\mathrm{i}}$. We can use this information to create a third-order approximation method that we believe has not yet appeared in the literature. As we will see in the next section, this method seems to perform significantly better than the first two in many cases. 
As in the Quadratic method, we try to fit a surface to the adjacent vertex data. Let

$$
f(x, y)=\frac{A}{2} x^{2}+B x y+\frac{C}{2} y^{2}+D x^{3}+E x^{2} y+F x y^{2}+G y^{3}
$$

The Weingarten matrix for this surface is still

$$
W=\left(\begin{array}{ll}
A & B \\
B & C
\end{array}\right)
$$

because in the local coordinatesystem the curvature only depends on the second-degreeterms. However, using the third degree terms in the least-squares fit will produce values for $(A, B, C)$ different from the ones we get in the quadratic method. The normal to this surface is given by:

$$
\begin{gathered}
N(x, y)=\left(f_{x}(x, y), f_{y}(x, y),-1\right) \\
=\left(A x+B y+3 D x^{2}+2 E x y+F y^{2}, B x+C y+E x^{2}+2 F x y+3 G y^{2},-1\right) .
\end{gathered}
$$

Let $\left(a_{i}, b_{i}, c_{i}\right)$ denote the normal at the data point $\left(x_{i}, y_{i}, z_{i}\right)$ (both normal and point must betransformed to the local coordinates), and let

$$
\mathbf{x}=\left(\begin{array}{lllllll}
A & B & C & D & E & F & G
\end{array}\right)^{\top} .
$$

Rewrite the normal as $\left(-\frac{a_{i}}{c_{i}},-\frac{b_{i}}{c_{1}},-1\right)$. Then, for each point, we have an equation

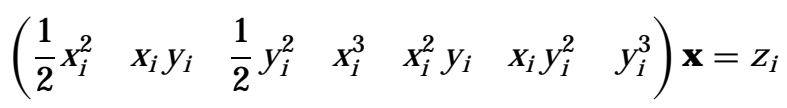

and for each normal we have two equations:

$$
\begin{aligned}
& \left(\begin{array}{lllllll}
x_{i} & y_{i} & 0 & 3 x_{i}^{2} & 2 x_{i} y_{i} & y_{i}^{2} & 0
\end{array}\right) \quad x=-\frac{a_{i}}{c_{i}} \\
& \left(\begin{array}{lllllll}
0 & x_{i} & y_{i} & 0 & x_{i}^{2} & 2 x_{i} y_{i} & 3 y_{i}^{2}
\end{array}\right) \quad x=-\frac{b_{i}}{c_{i}} .
\end{aligned}
$$

As in the preceding method, we scale these equations by the same scale factor $\frac{2}{\mathrm{k}_{i}^{2}}$, to obtain a system

$$
\mathrm{U} \mathbf{x}=\mathbf{d},
$$

where $U$ is a $3 n \times 7$ matrix and $\mathbf{d}$ is a $3 n$-vector. Again, we find a least-squares fit, but use only $A, B$, and $C$ from the result. Note that $n$ has to be at least 3 for there to be at least as many equations as unknowns.

\subsection{Higher Order Methods}

The preceding method can be extended to higher orders:

$$
\begin{gathered}
f_{4}(x, y)=\frac{A}{2} x^{2}+B x y+\frac{C}{2} y^{2}+\sum_{n=0}^{n=3} D_{n} x^{3-n} y^{n}+\sum_{n=0}^{n=4} E_{n} x^{4-n} y^{n} \\
f_{5}(x, y)=\frac{A}{2} x^{2}+B x y+\frac{C}{2} y^{2}+\sum_{n=0}^{n=3} D_{n} x^{3-n} y^{n}+\sum_{n=0}^{n=4} E_{n} x^{4-n} y^{n}+\sum_{n=0}^{n=5} F_{n} x^{5-n} y^{n}
\end{gathered}
$$

ACM Transactions on Graphics, Vol. 23, No. 1, J anuary 2004. 


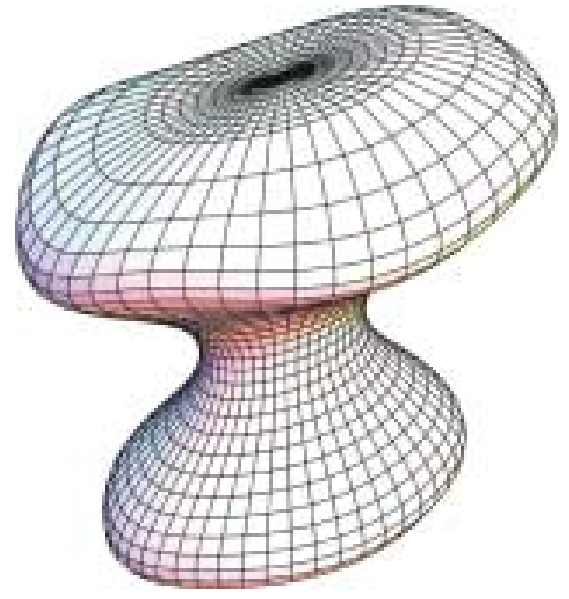

Fig. 1. Test Surface.

and so on. However, in order that there be at least as many equations as unknowns, the maximum degree of the approximation that we can compute is limited by the number of adjacent vertices in the mesh. For example, if we use 6 adjacent vertices, then there will be 18 equations. The highest order method that we can use is then degree 5 , since such an approximation will have the 18 unknowns $\left(A, B, C, D_{0}, \ldots, D_{3}, E_{0}, \ldots, E_{4}, F_{0}, \ldots, F_{5}\right)$.

As we will see in what follows, higher order methods seem to make small errors smaller, but can also make large errors larger.

\section{TESTING THE METHODS}

We set out to design a test surface (Figure 1) that had significant areas where the curvature was "interesting".

Our surface, $\mathrm{S}(\mathrm{u}, \mathrm{v})$, is defined by:

$$
\begin{gathered}
f(u)=-2 u^{4}+2 u^{2}+u / 6+0.3 \\
g(u)=\left\{\begin{array}{rr}
0.9+u+f(-0.9), & -0.9-f(-0.9) \leq u \leq-0.9 ; \\
f(u), & -0.9 \leq u \leq 1 ; \\
1-u-f(1), & 1 \leq u \leq 1+f(1) ;
\end{array}\right. \\
h(u)=\left\{\begin{array}{rr}
-0.9, & -0.9-f(-0.9) \leq u \leq-0.9 ; \\
\begin{array}{l}
u, \\
1,
\end{array} \quad \begin{array}{l}
1 \leq u \leq 1+f(1) ; \\
x(u, v)=g(u) \cos (v)
\end{array} \\
y(u, v)=g(u) \sin (v)
\end{array}\right. \\
S(u, v)=(x(u, v), y(u, v), z(u, v)) \quad 0.9-f(-0.9) \leq u \leq 1+f(1), \quad 0 \leq v \leq 2 \pi .
\end{gathered}
$$



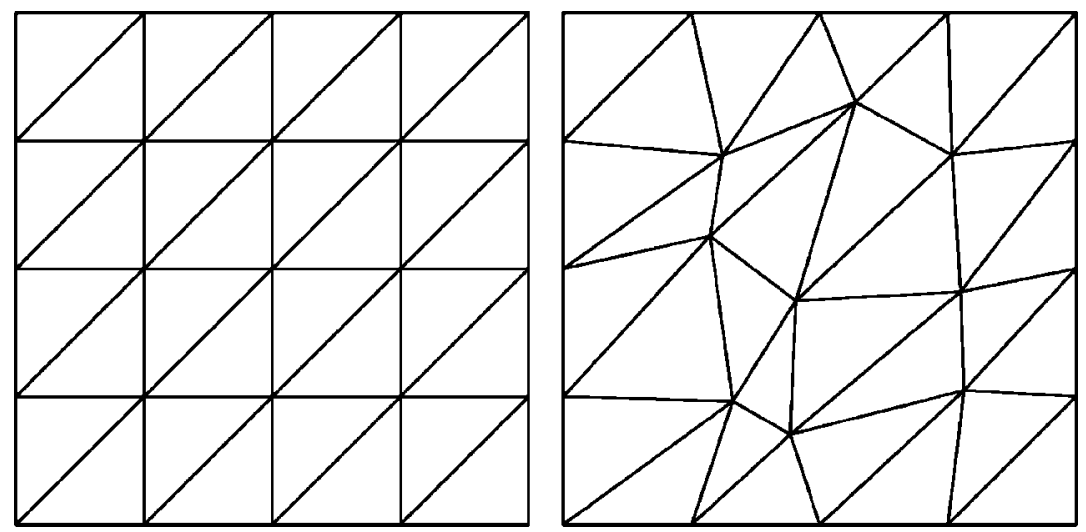

Fig. 2. Left: Regular Triangulation in parameter space, Right: Random Triangulation in parameter space.

\subsection{Global Parameter Space Triangulations}

For our first set of tests, we created two different gl obal mesh representations of the surface. First, we created a Global Regular triangulation of the parameter space. This is the type of meshing condition that we had found most commonly used for demonstrating the results of previous principal direction estimation algorithms. Figure 2-left shows an example of a 4-by-4 global regular triangulation. The actual triangulation we used was 50-by-50.

Then, we created a Global Random triangulation by jittering the locations of the interior points within the parameter space. This more closely resembles the type of meshing condition that we had encountered in practice, when desiring to estimate the principal directions at the vertices of arbitrary polygonally defined models. Figure 2-right shows an example of a 4-by-4 global random triangulation in parameter space.

By definition, all of the object space vertices obtained from each of these parameter space triangulations lay exactly in the analytically defined surface.

Figure 3 shows the results of estimating principal directions using each of thethree principal direction approximation methods described in Section 3, in the case where the data is represented by the Global Regular mesh. All of the methods perform very well under this condition.

Figure 4 shows the estimated principal directions computed using the same three methods at the vertices of the Global Random mesh. In this case, we found a dramatic increase in the prevalence of large angle errors in the principal direction estimates, even at points that were far from being umbilic. The increase in errors is especially severe with the two second-order methods. Estimated directions more than $10^{\circ}$ out of alignment with the true principal directions are highlighted in red.

\subsection{Local Tangent Space Projections}

Seeking deeper insight into the source of the large errors in principal direction estimates under nonregular meshing conditions, we set out to devise a number of different local triangular meshes on the surface that we could use to investigate the following questions:

(1) Does regularity of the mesh improve accuracy and randomness reduce it?

(2) Does enforcing equiangularity between adjacent vertices improve accuracy?

(3) Does enforcing equidistance to adjacent vertices improve accuracy?

(4) Does choosing adjacent vertices that lie along the true principal directions have any effect on accuracy? 

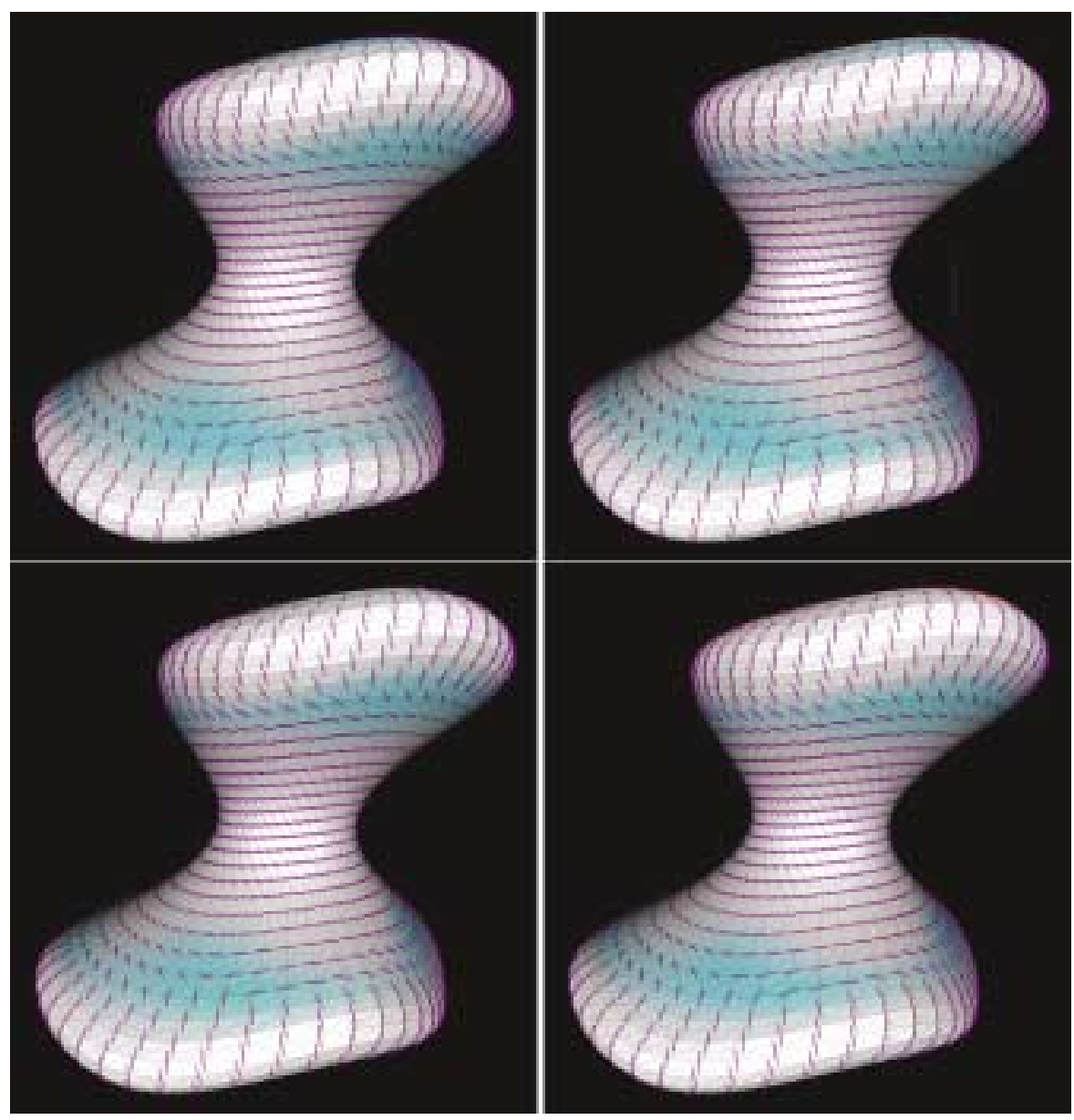

Fig. 3. Approximated principal directions, Global Regular mesh. Directions marked in red have errors above $10^{\circ}$. Surface coloration highlights areas in which the difference between the two principal curvatures becomes small (near-umbilic points). Upper left: exact directions. Upper right: approximated directions, Quadratic Method. Lower left: approximated directions, Normal Curvature Method. Lower right: approximated directions, Cubic Method.

With these in mind, we created six local meshing patterns, which we defined independently around each of 575 individual vertices randomly sampled from the global random mesh. None of the 575 local mesh centerpoints were umbilical points. Roughly $80 \%$ satisfied $\lambda_{1}-\lambda_{2}>0.5$; the remainder had $\lambda_{1}-\lambda_{2}$ reasonably uniformly distributed between 0 and 0.5 .

To obtain the local surface meshes, we began by defining, in parameter space, the six planar mesh patterns shown in Figure 5. We achieved each of these patterns by rotating a single radial edge about a given centerpoint according to various criteria. I $n$ all cases, a total of six edges were defined, with the first edge aligned with the analytically determined first principal direction. For the first local mesh, we used a constant angle of rotation $\left(60^{\circ}\right)$ to define all of the edges. With this mesh we would be able to test whether enforcing equiangularity between adjacent vertices improves the accuracy of the principal directions estimated at the central point. We refer to this mesh type as "local equi-angle". In the second case, we defined the angles between the edges to lie in a regular pattern with 3-way symmetry, by 

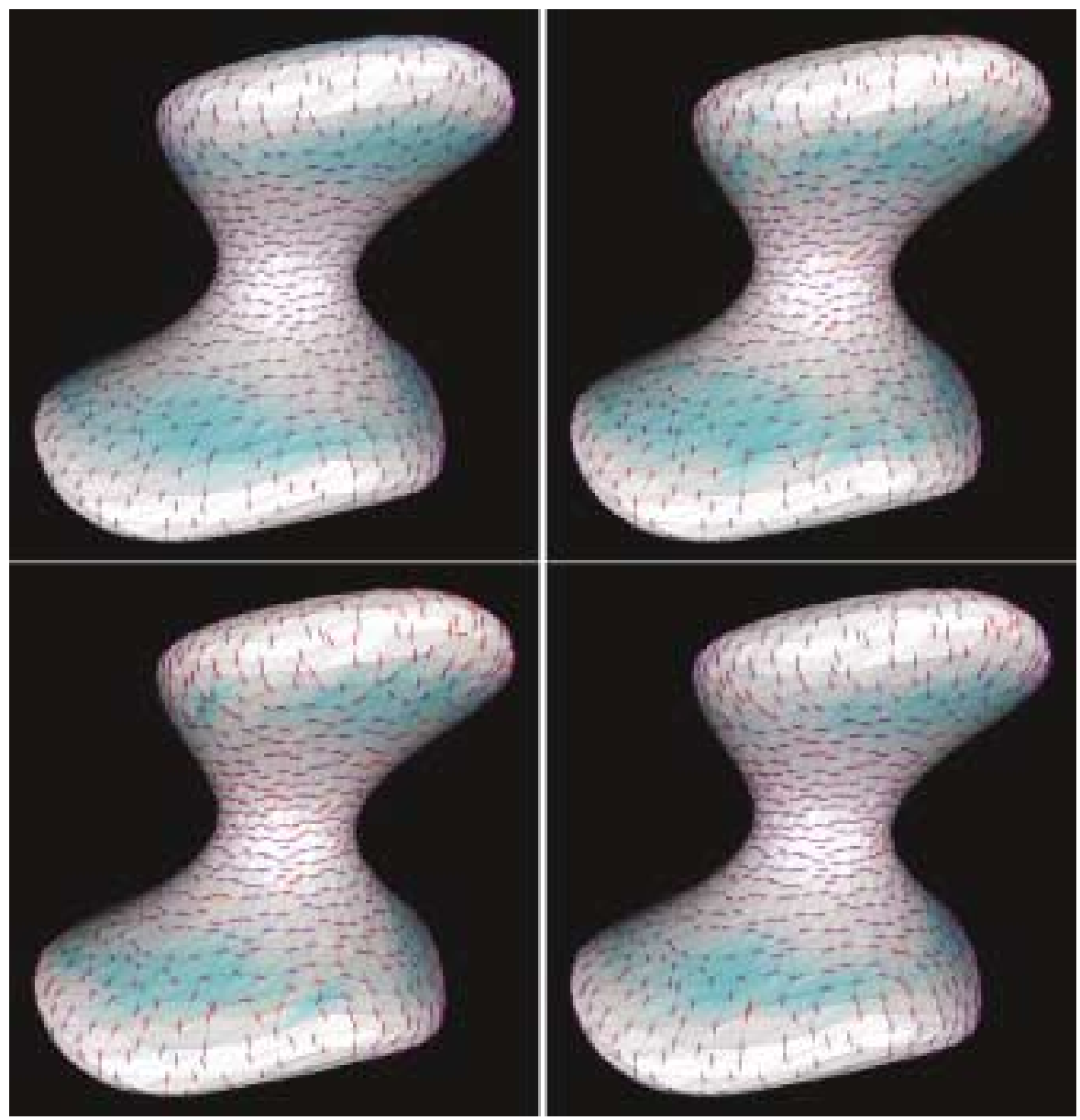

Fig. 4. Approximated principal directions, Global Random mesh. Directions marked in red have errors above $10^{\circ}$. Surface coloration highlights areas in which the difference between the two principal curvatures becomes small (near-umbilic points). Upper left: exact directions. Upper right: approximated directions, Quadratic Method. Lower left: approximated directions, Normal Curvature Method. Lower right: approximated directions, Cubic Method.

alternating $30^{\circ}$ with $90^{\circ}$ rotations. We chose this pattern in order to test the question of whether mesh 'regularity', in terms of a certain symmetry in the locations of edges, improves the accuracy of the results. We refer to this mesh type as "local 30-90". In the remaining four cases we defined the meshes by randomly choosing five successive angles of rotation in $\left[1^{\circ} . .90^{\circ}\right]$. We refer to these meshes as "local random [1-4]". We felt that it was important to additionally test the methods on a reasonable set of randomly defined local meshes in order to establish a larger context within which to interpret the results we would find in the more highly constrained conditions.

For each of the six local mesh patterns in parameter space we obtained a collection of 575 local surface meshes by determining the points on our analytically defined test surface that projected onto each pattern when the pattern was laid in the tangent plane to the surface at each of the 575 sample points (Figure 6).

Finally, we rotated each of the patterns in the tangent plane 5 times ( 72 degrees each time). We did this in order to investigate the hypothesis that having some mesh edges coincidentally aligned with or 

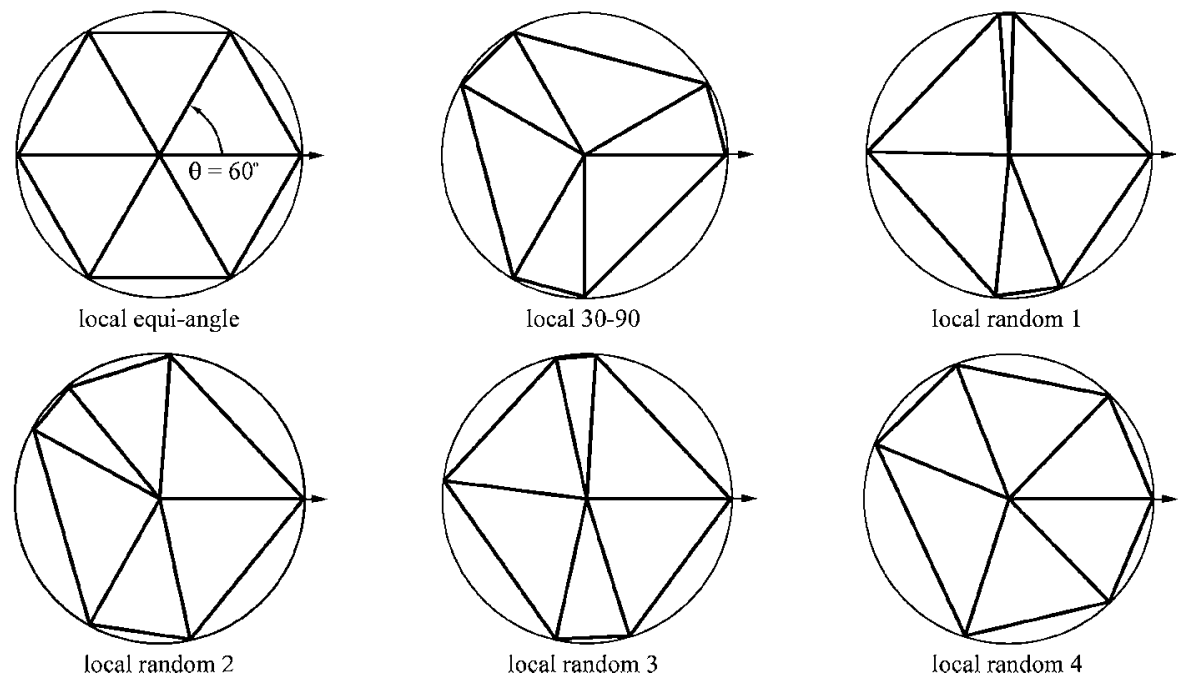

Fig. 5. Our six local mesh patterns in parameter space.

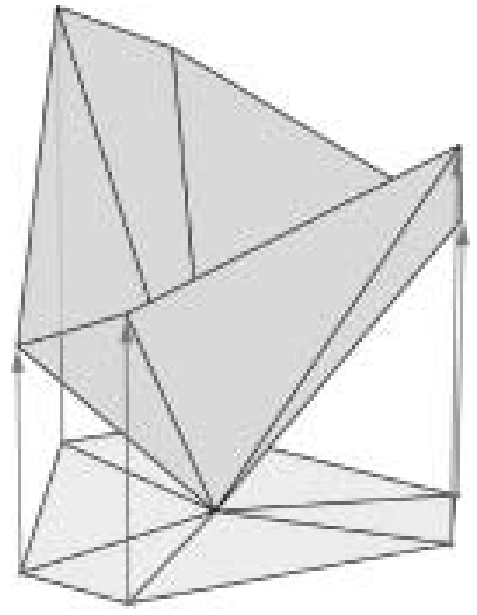

Fig. 6. How a local surface mesh is obtained from a local mesh template in the tangent plane.

counterbalanced against the principal directions might affect the accuracy of the approximation. The initial orientation of each local mesh was configured to have one edge lying along a principal direction. Subsequent rotations moved this edge away from the principal direction.

\subsection{Findings and Interpretation}

The results of our further testing are summarized in Figures 7 and 8. Detailed tables are available on the ACM TOG website, www.acm.org/pubs/tog.

Figure 7 shows the median angle error, in degrees, obtained with each of the three principal direction estimation methods, applied both to the two gl obal meshes and to each of the six different local meshes 


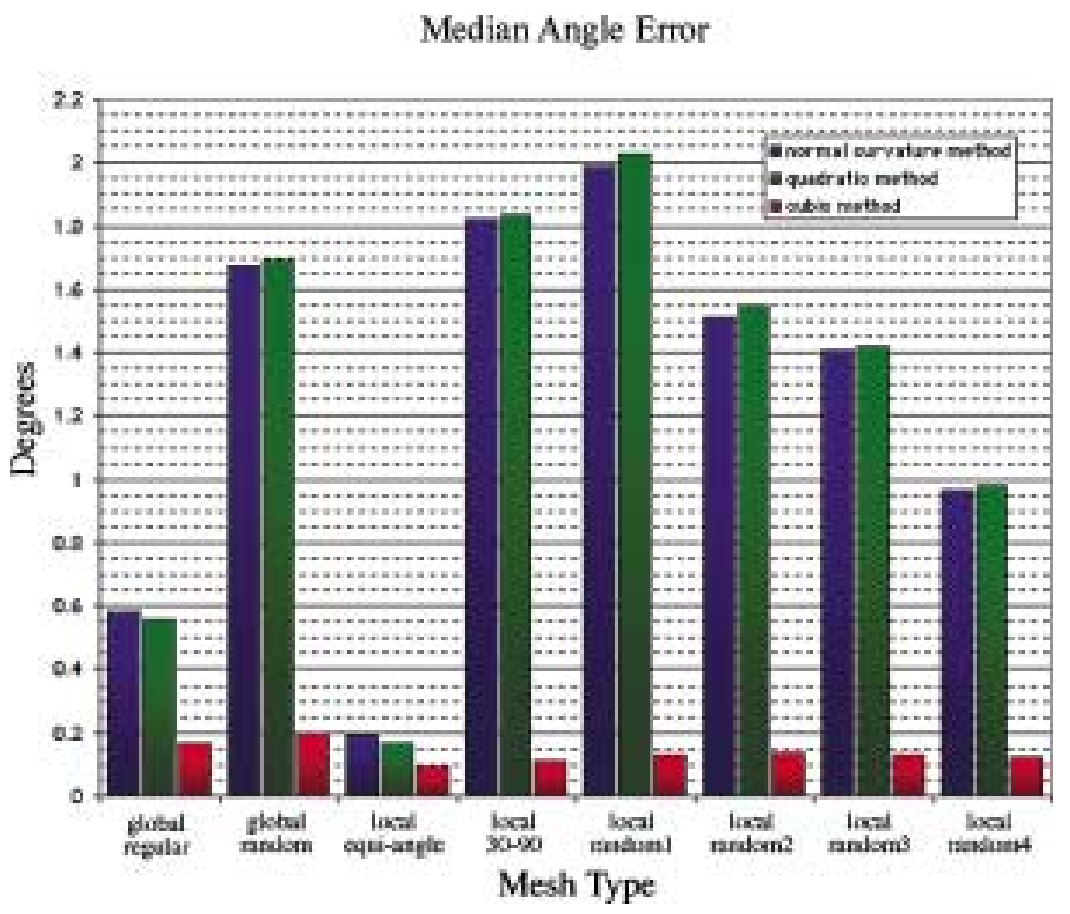

Fig. 7. Median angle error in the approximated principal directions, per mesh type and method.

Cubic Method

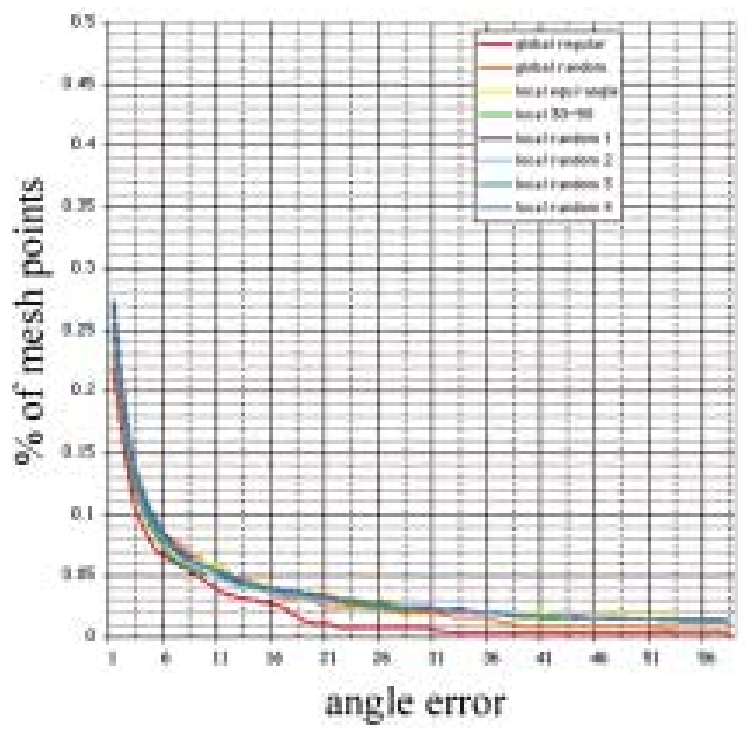

Normal Curvature Method

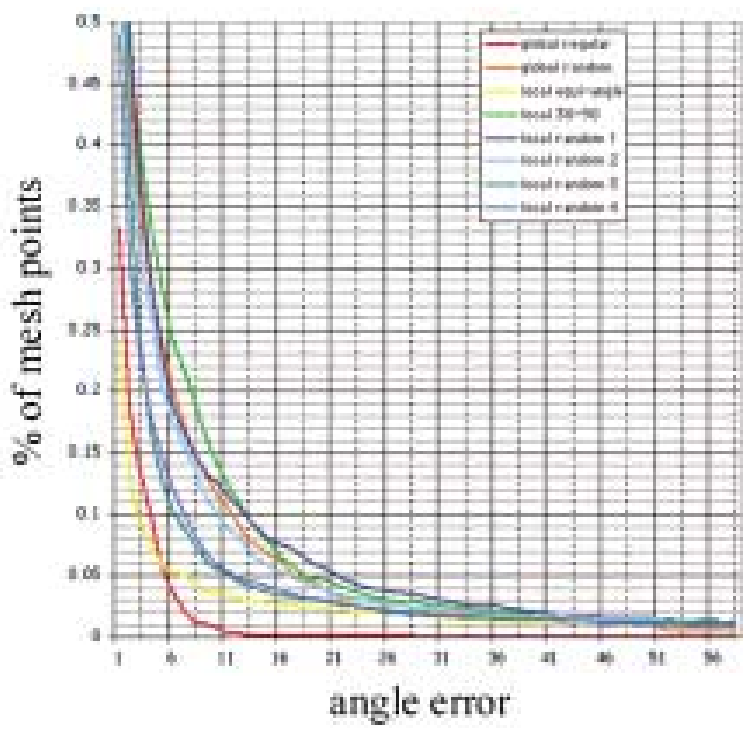

Fig. 8. Prevalence of errors in the approximated principal directions, by magnitude. 

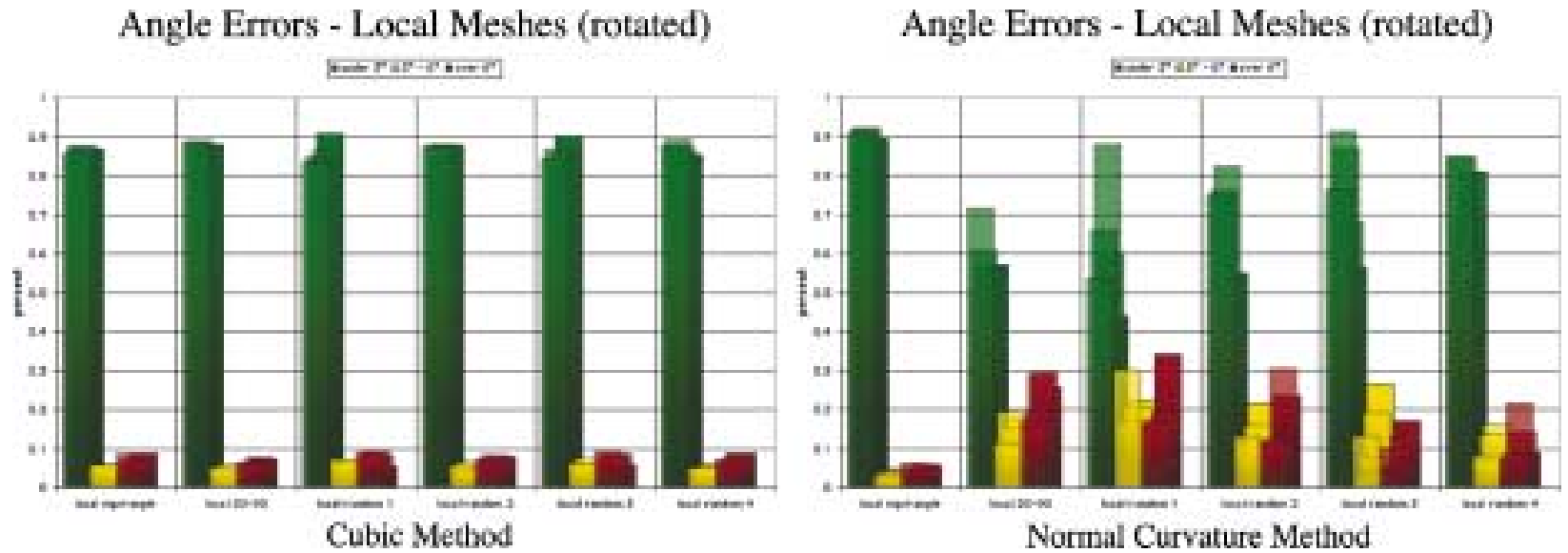

Fig. 9. Distribution of errors in the approximated principal directions, found using five different rotations of the local meshes with each of the six local meshing schemes. Results are quantized into three ranges: less than $3^{\circ}$ (green), between $3^{\circ}-6^{\circ}$ (yellow), and more than $6^{\circ}$ (red). The graphs representing each of the different rotation conditions are layered in depth, with the zero rotation condition appearing at the rear.

at 575 sampled points on the surface. Median errors for the local mesh results were computed using data from all five mesh rotations, or 2875 values in all.

Overall, performance was best when the Cubic method was used, and was not as good in the cases of the Quadratic and Normal Curvature methods. The performance in the cases of the two second-order methods was very similar. The Cubic method exhibited the least variation in performance over the different mesh conditions. With the two second order methods, the size and prevalence of errors varied significantly according to themesh type, indicating that some meshing arrangements were "better" than others. The smallest errors occured in the local equi-angle and global regular cases, and the largest errors occured in the cases of the global and local random meshes and the local $30^{\circ}-90^{\circ}$ pattern.

Figure 8 provides a more detailed look at the data for the normal curvature and cubic methods, showing the percentage of mesh points at which the angle errors of the estimated principal directions fell below specific thresholds.

In Figure 8-left, we can again see that with the Cubic method, performance was fairly consistent across all mesh types. Approximately $22-27 \%$ of the mesh points had angle errors above $1^{\circ}$, with that number rapidly dropping to $5 \%$ with errors above $10^{\circ}-15^{\circ}$ and below $3 \%$ with errors above $20^{\circ}$, for all mesh types. In Figure 8-right, we can see again that, in the case of the second-order method, there is greater variation in performace under the different mesh conditions. In aggregate, between $23 \%$ to over $50 \%$ of the mesh points had angle errors above $1^{\circ}$, with that number dropping to $5 \%$ at thresholds between $5^{\circ}-22^{\circ}$, depending on mesh type. For this method, performance was particulary good in the cases of the global regular mesh, where fewer than $1 \%$ of mesh points had errors above $10^{\circ}$, and the local equi-angle mesh, where $90 \%$ of mesh points had errors below about $3^{\circ}$, but poorer in the other mesh conditions.

Table 1, provided on the ACM Web site, contains numeric data that lists, for each meshing scheme and each local mesh rotation, the number of points at which the degree error falls into each of three ranges: less than $3^{\circ}$, between $3^{\circ}-6^{\circ}$, and more than $6^{\circ}$.

Figure 9, derived from Table 1, illustrates the range of variation in angle errors found under the different mesh rotation conditions, for the cubic and normal curvature methods. Wenoticed no apparent advantage for the principal-direction aligned rotations. 
Figure 10, also derived from Table 1, illustrates the effects of using higher order methods. The results are not qual itatively different from the cubic case. Overall, accuracy is good with these methods, at most points, but high errors remain at a significant fraction of the others.

Figure 11, derived from Table 2 (on the ACM Web site), shows that the results are virtually identical when the constraint of equal edge length on the surface (as opposed to on the local parameterization in the tangent plane) is individually enforced for each local mesh. The local edge length was defined to be equal to the average of the lengths of the edges that had comprised the local mesh in the unequal edge length condition.

Not shown here, but available in Table 3 on the ACM Web site, are the results of further investigations in which we applied a globally equal edge length constraint, using two different edge lengths. As expected, we found that errors increased with increasing edge length. However, the overall pattern of performance across the different meshes and methods remained qualitatively similar to what was found in the local equal (and unequal) edge length conditions.

\subsection{The Effect of Errors in Surface Normal Approximations}

Up to this point, we have been examining the performance of the various principal direction estimation algorithms using analytically defined (error-free) surface normals. In practice, of course, the true surface normal cannot be exactly determined, and it is useful to consider how errors in the surface normal computations can affect the accuracy of principal direction estimates achieved using each of the previously considered methods.

As several different surface normal approximation methods have been proposed, we decided to take a closer look at three:

(1) The normalized average of the surrounding triangle unit normals.

(2) The normalized average of the surrounding triangle unit normals, each weighted by the angle of the triangle at $p$.

(3) The normalized average of the adjacent edges, each weighted by the sum of the cotangents of the angles opposite the edge in the two triangles sharing the edge [Meyer et al. 2003]. This weighting only makes sense if each pair of triangles is acute, so another method must be used at vertices where a triangle is not acute.

We experimented with each of these schemes on our test surface, using the six local surface meshes described above, at the same 575 randomly chosen sample points. In all cases, the vertices that we used lay in the analytically defined surface. Under these conditions, all of the normal approximation methods worked very well. The average error in the approximated normal over all mesh types was 0.90 degrees for the unweighted method, 0.97 degrees for the angle-weighted method, and 1.04 degrees for the edgelength-weighted method. The median error over all mesh types was 0.41 degrees for the unweighted method, 0.50 degrees for the angle-weighted method, and 0.54 degrees for the edgel engthweighted method. Although the normal vector approximation errors in the tabulated data appeared to be superficially slightly smaller for the angle-weighted method in the cases of the global meshes and for the unweighted method in the cases of the local meshes, we do not believe that these differences are significant either practically or statistically.

Figure 12, derived from Table 4 (on the ACM Web site), summarizes the results of using approximated rather than exact surface normals in the principal direction estimation calculations for all three methods, pooling results from all five local mesh rotations. The approximated normals at each point were obtained by taking the normalized average of the surrounding triangle unit normals.

The main effect, which can be seen even more clearly in the tabulated data, seems to be an increase the amount of volatility in the accuracy of the estimates produced by the second order methods (for 

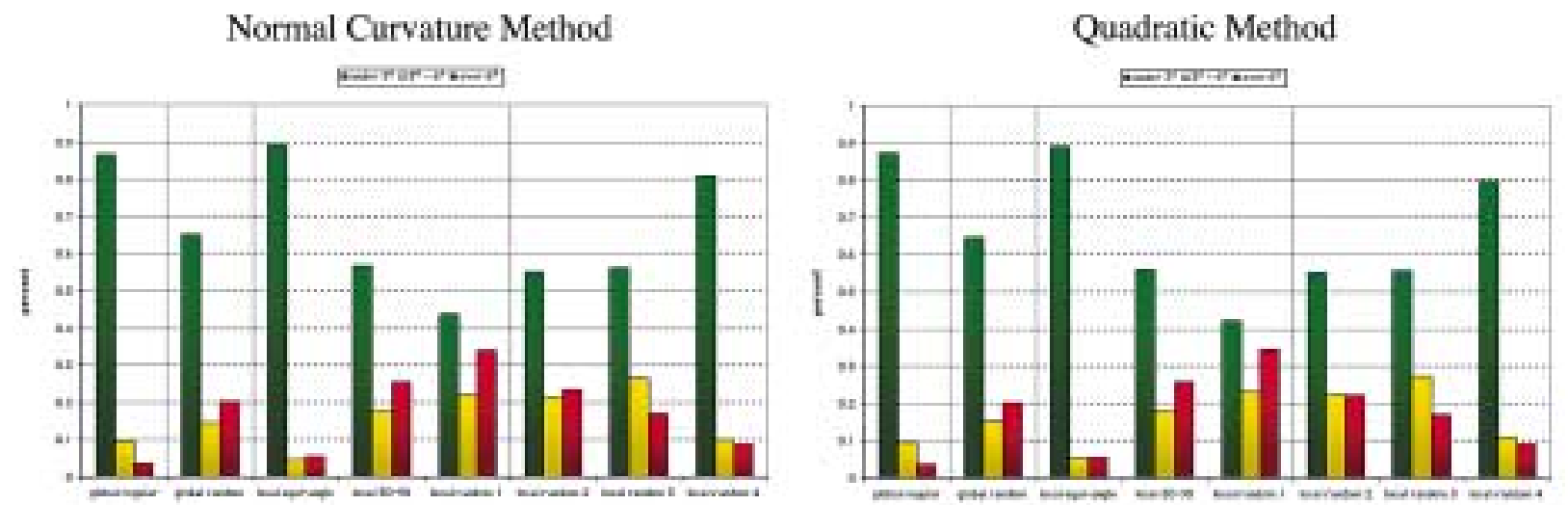

\section{Cubic Method}

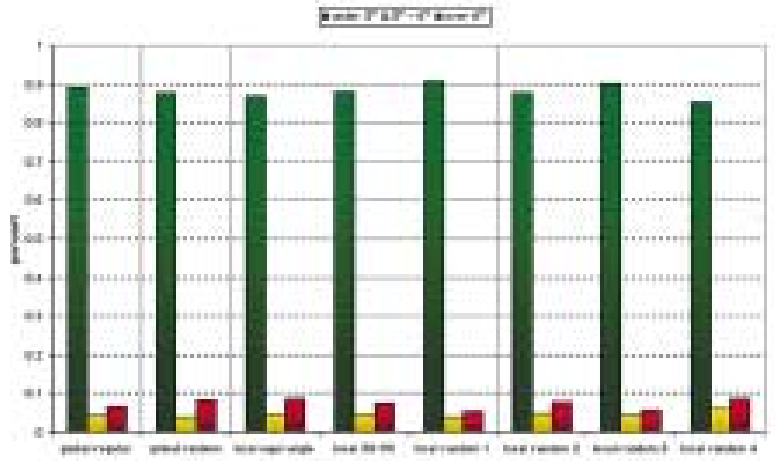

Fourth-order Method

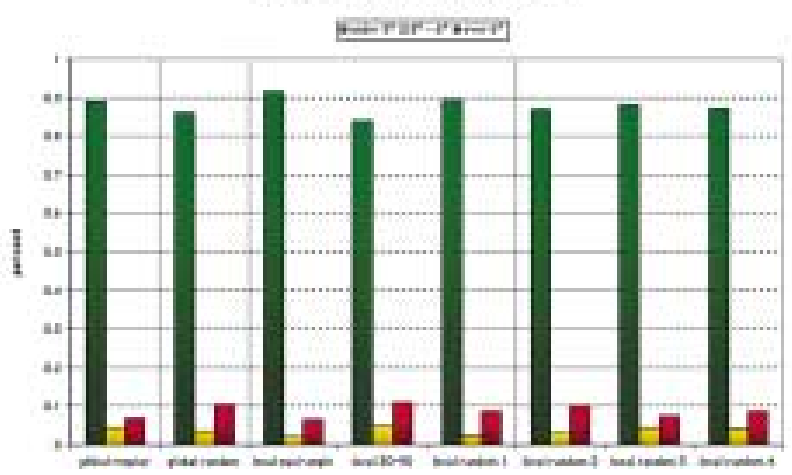

Fifth-order Method

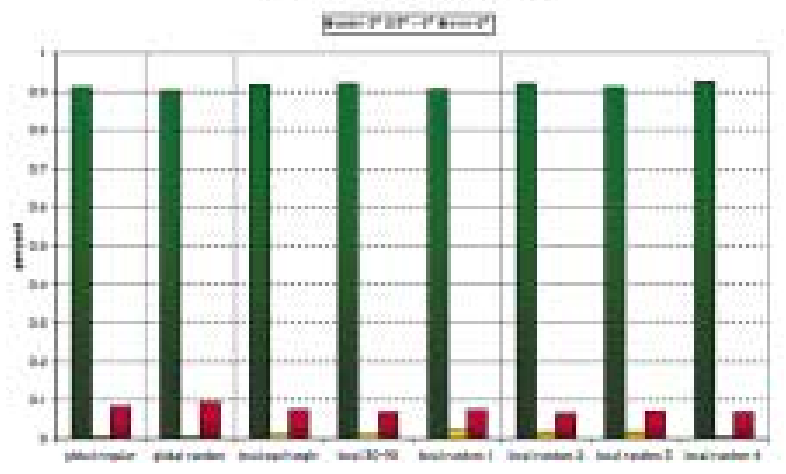

Fig. 10. A comparison of the errors found in principal directions approximated using second-through fifth-order methods. 
Equal/Unequal Local Edge Lengths

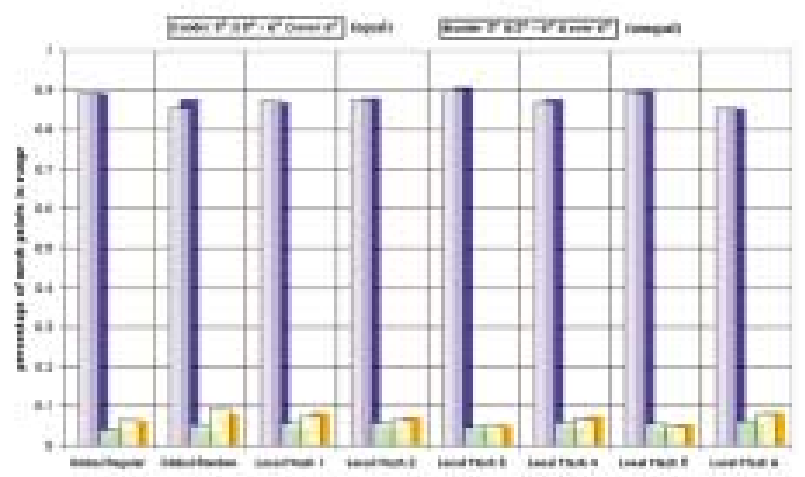

Cubic Męthod
Equal/Unequal Local Edge Lengths

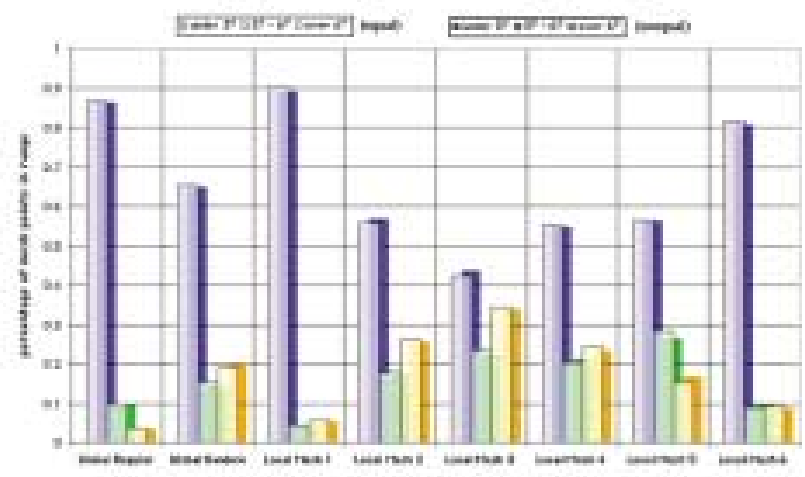

Normal Curvature Method

Fig. 11. Distribution of errors in the approximated principal directions when edges in each local surface mesh are constrained to be of equal length in object space (foreground), as opposed to being equal length in parameter space (background).

Approximate vs Exact Normals

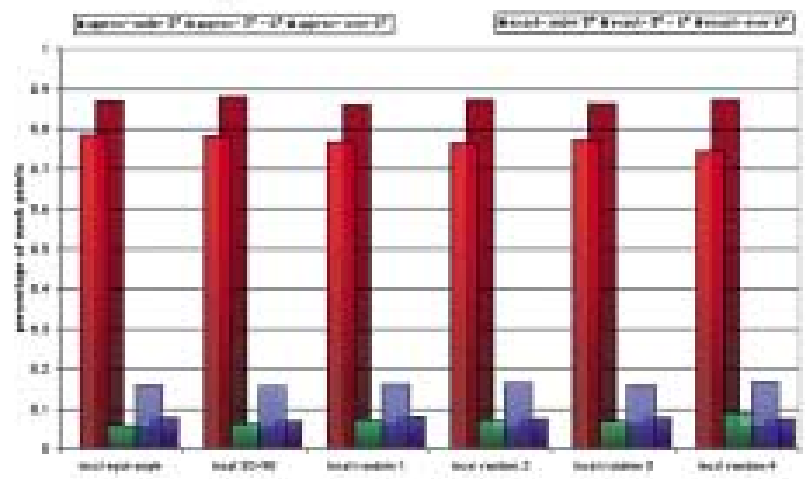

Cubs Method
Approximate vs Exact Normals

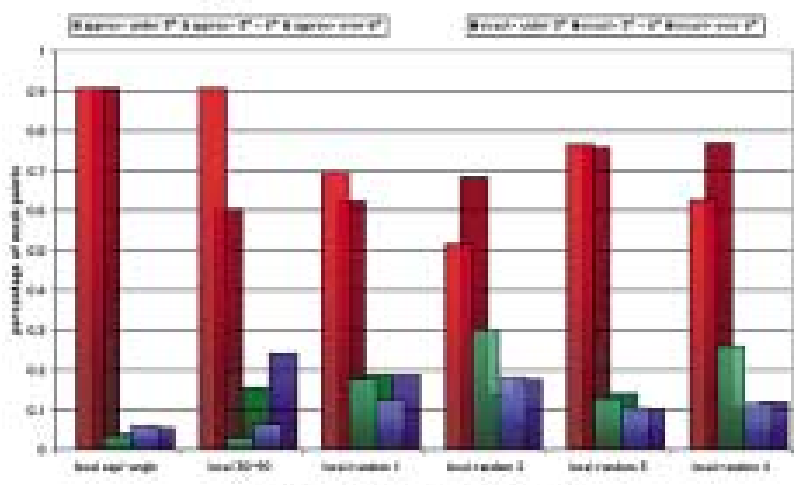

Nomnal Curvature Method

Fig. 12. Distribution of errors in the approximated principal directions when computations are performed using approximated, as compared to exact, values for the surface normals. The results with the approximated normals are in the lighter colors, in the foreground, with the results using exact normals given in a background layer for reference.

some meshes, in some rotations, resulting in greater errors, and for other meshes, or other rotations, resulting in fewer). Overall accuracy appears to be particularly good, for these methods, when the the approximated normals are used in combination with the two "regular" local mesh schemes-the local equi-angle and the local 30-90, and not so good under the other mesh conditions. Consistent with our findings that the third and higher order methods appear to be less sensitive to variations in the local mesh characteristics, the impact on the accuracy of the estimates produced by the Cubic Method is more muted. There is a small but consistent downward trend in accuracy with the use of the approximated, rather than exact surface normal values, with the Cubic M ethod. However, overall performance remains good, with over 75 having errors of less than $3^{\circ}$ and fewer than $20 \%$ having errors of greater than $6^{\circ}$.

In a last set of experiments, detailed in Table 5, weinvestigated theimpact of reducing the val ence at a vertex from 6 to 3 . Wecomputed this data for three different local meshes at 625 randomly sel ected points on a torus dataset. We also computed local meshes with valences 5 and 4 at the previously mentioned 
575 selected points on the parametric surface dataset, and obtained similar results. We found, as might be expected, that the accuracy of the results produced by all three methods decreases as the valence drops, practically plummeting, for some meshes and methods, as the number of participating edges falls to 3. However, the situation is rarely worse in the case of the cubic method than it is when either of the secod order methods is used.

Finally, we reexamined the our principal direction error findings considering all potentially "near umbilic" sample points, defined as having with a principal curvature difference of less than 0.2 , separately from the rest of the 575 points. In Figure 13, we highlight the distribution of errors at these points in red, within the overall distribution. This explicitly reveals that large principal direction errors can occur away from umbilical points.

In sum, our results suggest several conclusions:

(1) The degree $\geq 3$ methods nearly consistently outperform both of the degree 2 methods.

(2) The regular meshes tend to produce better results than the random ones.

(3) The equiangular mesh (Local Mesh 1) produces good results for all three methods.

(4) Aligning edges with principal directions does not seem to matter.

(5) Enforcing equality of adjacent edge length does not seem to matter, but the shorter all of the edges are the better.

(6) Large errors can occur away from umbilical points.

After further mathematical investigation into potential sources of the errors encountered, we determined that in the case of the cubic method, the sizes of the errors in the estimates of the normal curvatures seems to be the factor most significantly influencing the size of the error in the principal direction estimates. In the case of the normal curvature method, the direction in which the errors were made seems, for some meshes, to be nearly as significant. In other words, the accuracy of the normal curvature method may be more dependent on the incidence of situations in which errors are able to cancel out.

\section{CONCLUSIONS}

We have shown that approximating principal directions on a surface mesh can be a tricky business. Although it is important to minimize the error in approximating various quantities such as normal curvature, an unfortunate pattern of small errors can produce large angle errors. Using a nontrivial test surface, we have shown that principal direction approximation methods are particularly prone to error when working with irregularly sampled data, and that these errors appear to be more severe for second order methods than for higher order methods that can take advantage of more of the known information about the surface.

There are many important directions for future work in this area. A major practical concern that we did not touch upon at all in our work is the problem of surface smoothing. In many cases, approximate meshes are obtained from a sampling process that is prone to a certain amount of intrinsic error. How might we best recover the mesh that describes the continuous smooth surface that is approximated by a collection of noisy samples? If we have only a mesh that is highly irregular as well as noisy, how could we improve our chances of obtaining a smooth principal direction vector field and avoiding theintroduction of large principal direction estimation errors? Would resampling to obtain a mesh in which the edge distribution is more equiangular help? Finally, it would be interesting to see how well the findings we have made hold up under further testing with a wider variety of mesh types and surface shapes. 
Normal Curvature Method

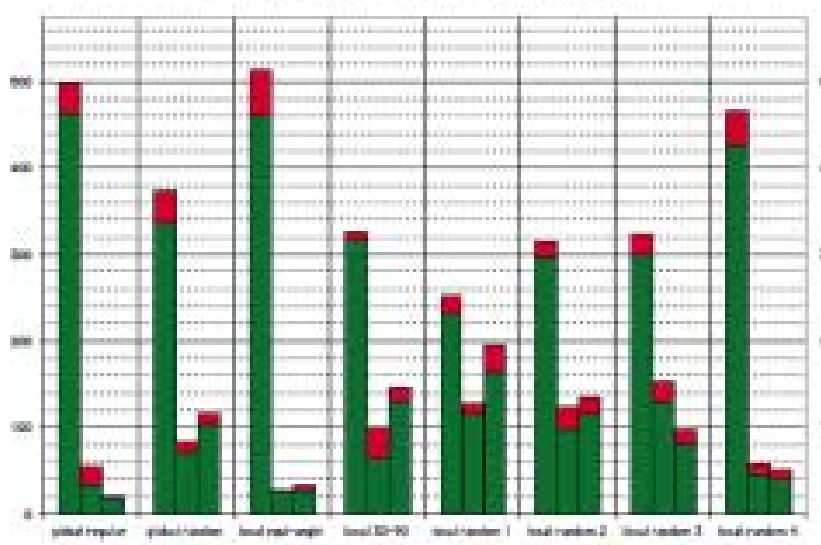

Quadratic Method

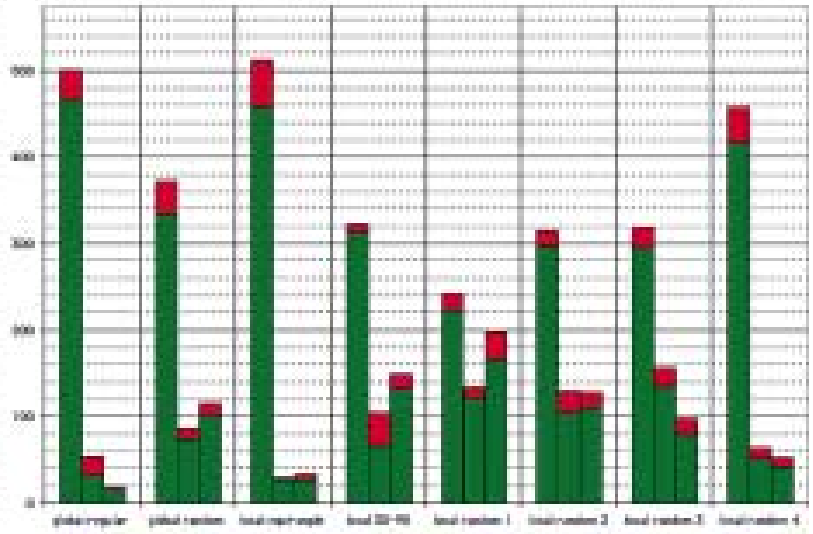

Cubic Method

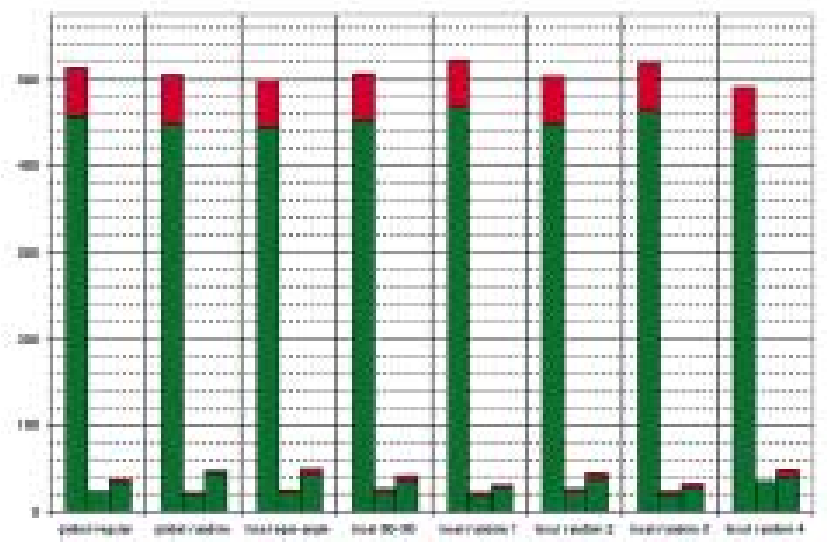

Fourth Order Method

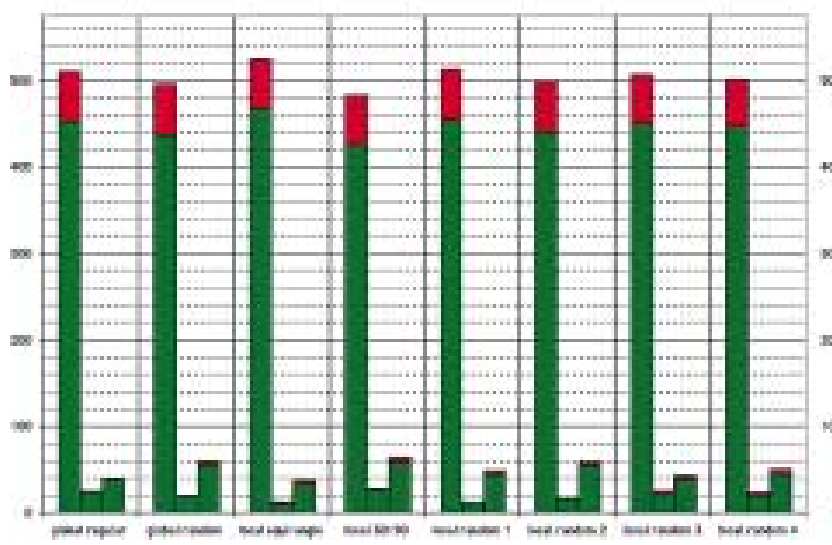

Fifth Order Method

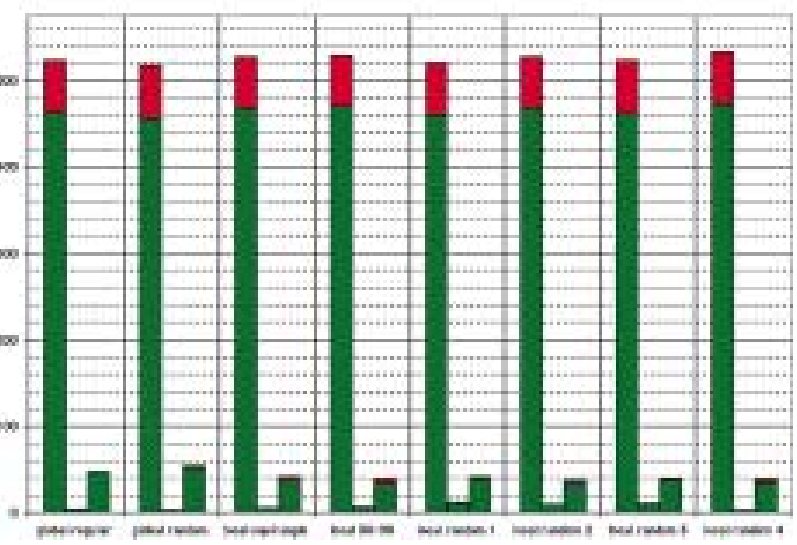

Fig. 13. Histogram distribution of the angle error in the principal directions estimated using the five different methods and eight different mesh configurations discussed above, with the results due to "near umbilic" points highlighted in red. Bin ranges: $\left\{<3^{\circ}, 3^{\circ}-6^{\circ},>6^{\circ}\right\}$

ACM Transactions on Graphics, Vol. 23, No. 1, J anuary 2004. 


\section{REFERENCES}

Chen, X. And Schmitt, F. 1992. Intrinsic surface properties from surface triangulation. In Proceedings, European Conference on Computer Vision. 739-743.

Diewald, U., Preußer, T., and Rumpf, M. 2000. Anisotropic diffusion in vector field visualization on eudidean domains and surfaces. IEEE Trans. Visual. Comput. Graph. 6, 1 (Apr./J une), 139-149.

Duncan, B. And Olson, A. 1992. Shape analysis of protein surfaces. J. Mol. Graph. 10, 1 (Mar.), 50.

FLYNN, P. J. AND J AIN, A. K. 1989. On reliable curvature estimation. In Proceedings of the IEEE Computer Society Conference on Computer Vision and Pattern Recognition. 110-116.

Girshick, A., Interrante, V., Haker, S., and Lemoine, T. 2000. Line direction matters: an argument for the use of principal directions in line drawings. In Proceedings of the 1st I nternational Symposium on Non Photorealistic Animation and Rendering (Annecy), 43-52.

Gorla, G., Interrante, V., and Sapiro, G. 2003. Texture synthesis for 3d shape representation. IEEE Trans. Visual. Comput. Graph. 9, 4 (Oct./Dec.), 512-524.

HahmANn, S. 1999. Visualization techniques for surface analysis. In Advanced Visualization Techniques, C. Bajaj, Ed. Wiley, New York.

Hamann, B. 1994. Curvature approximation of 3d manifolds in 4d space. Comput. Aid. Geomet. Des. 11, 6, 621-632.

Hertzmann, A. ANd Zorin, D. 2000. Illustrating smooth surfaces. In Siggraph 2000, Computer Graphics Proceedings, K. Akeley, Ed. ACM Press/ACM SIGGRAPH/Addison Wesley Longman, 517-526.

INTERRANTE, V. L. 1997. Illustrating surface shape in volume data via principal direction-driven 3D line integral convolution. Comput. Graph. 31, Annual Conference Series, 109-116.

Meyer, M., Desbrun, M., Schröder, P., and Barr, A. H. 2003. Discrete differential-geometry operators for triangulated 2manifolds. In Visualization and Mathematics III, H.-C. Hege and K. Polthier, Eds. Springer-Verlag, Heidel berg, Germany, 35-57.

Samson, P. and Mallet, J.-L. 1997. Curvature analysis of triangulated surfaces in structural geology. Math. Geol. 29, 3 (Apr.), 391-412.

Takashi Maekawa, F.-E. W., and Patrikalakis, N. M. 1996. Umbilics and lines of curvature for shape interrogation. Comput. Aid. Geomet. Des. 13, 2 (Mar.), 133-161.

TAUBIN, G. 1995. Estimating the tensor of curvature of a surface from a polyhedral approximation. In Proceedings of the 5th International Conference on Computer Vision. 902-907.

Received September 2001; revised J une 2003; accepted J uly 2003 